\title{
Génétique des textes et génétique des cycles : pourquoi changer d'échelle?
}

\section{Olivier Lumbroso}

\section{(2) OpenEdition}

1 Journals

\section{Édition électronique}

URL : https://journals.openedition.org/genesis/1594

DOI : 10.4000/genesis. 1594

ISSN : 2268-1590

\section{Éditeur :}

Presses universitaires de Paris Sorbonne (PUPS), Société internationale de génétique artistique littéraire et scientifique (SIGALES)

\section{Édition imprimée}

Date de publication : 9 mai 2016

Pagination : 19-36

ISBN : 9791023105315

ISSN : 1167-5101

\section{Référence électronique}

Olivier Lumbroso, «Génétique des textes et génétique des cycles : pourquoi changer d'échelle? », Genesis [En ligne], 42 | 2016, mis en ligne le 05 juin 2017, consulté le 17 janvier 2023. URL : http:// journals.openedition.org/genesis/1594; DOI : https://doi.org/10.4000/genesis.1594 


\section{Génétique des textes et génétique des cycles : pourquoi changer d'échelle?}

Olivier Lumbroso

Les plus belles âmes sont celles qui ont plus de variété et de souplesse*.

I 1 serait utile de fonder une critique génétique adaptée aux cycles, comme il existe une génétique des textes. Cette dernière a franchi les échelles : de la variante à l'approche des réécritures outillées par la linguistique, de la génétique stylisticienne à celle du romanesque, décrite par Henri Mitterand, qui a ouvert les chemins de la génétique du scénario ${ }^{1}$. Dans la continuité d'un tel élargissement, verrait le jour une génétique appropriée aux agencements cycliques, à savoir aux œuvres qui entretiennent entre elles des liens de solidarité organique, combinant, selon les cas, la circularité et le bouclage, le retour et la reprise, l'esprit totalisateur et unificateur d'un grand tout qui se déploie dans la durée. Qu'ils soient, comme le dit Christophe Pradeau, à entrée «unique», tel que le roman-fleuve imposant la continuité d'une histoire collective qui se déroule linéairement, tome après tome, ou qu'ils soient à entrée «multiple ${ }^{2}$ », tel que le cycle zolien et balzacien, reposant sur la circularité, la reprise ou le bouclage au sein de romans autonomes et distincts sur les plans des contenus et de la forme, ces monuments littéraires s'épanouissent dans la durée, décrochent du modèle rectiligne, et supportent le tumulte de l'Histoire. Dans l' «Entretien» que publie le numéro 36 de Genesis, consacré à «Proust, 1913», Bernard de Fallois souligne que l'histoire d'un roman est un roman. L'histoire d'un cycle, pourrait-on dire, est l'aventure d'une écriture qui fait le pari fragile de s'auto-engendrer, en envisageant de façon plus ou moins optimiste sa longévité. Comme le souligne Jules Romains, dans son projet de préface aux Hommes de bonne volonté, évitons la ruine de l'opus magnum : "Quand le huitième - qui sait? - le dixième se présenteront, y aura-t-il encore une "époque" pour les recevoir, ou bien est-ce que tout aura sauté3 ${ }^{\text {? } » ~ L a ~ r e ́ f e ́ r e n c e ~ i m p l i c i t e ~ a ̀ ~ l a ~ G r a n d e ~ G u e r r e ~ d e ~ 1914-1918, ~ q u i ~ r e s t e ~}$ douloureuse chez l'auteur du roman-fleuve, trahit un traumatisme de l'Histoire qui fragilise la confiance de l'écrivain. Cette conception diffère du statut de la guerre franco-prussienne, dont Zola fait la pierre angulaire de la composition «bouclée» du cycle consacré au Second Empire, dès le lancement de celui-ci en 1870, lors de la publication de La Fortune des Rougon ${ }^{4}$. De l'un à l'autre, de l'architecture maçonne au risque de délitement, le sens et la

\footnotetext{
* Montaigne, Les Essais, éd. Pierre Villey, Paris, PUF, 1988, Livre III, p. 818.

1. Voir l' «Avant-propos» d'H. Mitterand à l'ouvrage Leçons d'écriture : ce que disent les manuscrits, dir. Almuth Grésillon et Michael Werner en hommage à Louis Hay, Paris, Minard, 1985. Voir les actes du colloque de 1999 édités par J.-P. Leduc-Adine : Zola. Genèse de l'œuvre, Paris, Éditions du CNRS, 2002.

2. Sur cette distinction, je renvoie à l'article de Christophe Pradeau dans le présent volume de Genesis.

3. Jules Romains, Les Dossiers préparatoires des «Hommes de bonne volonté», présenté par A. Angremy, Cahiers Jules Romains, Paris, Flammarion, 1983, t. V, p. 191.

4. Sur la distinction du cycle et du roman-fleuve en lien avec l'Histoire et la Grande Guerre, je renvoie à l'article d'Aude Leblond dans le présent volume de Genesis.
} 
valeur de la composition cyclique changent de sens et revisitent le genre, façon de féconder, en les dépassant, les héritages de Balzac et Zola, deux écrivains confiants dans les taxinomies et les inventaires encyclopédiques.

Cette génétique des cycles serait-elle alors capable de sonder le laboratoire qui produit ces formes distendues, sensibles aux vibrations de l'Histoire? Étudier le programme fondateur et les mécanismes de cyclisation poétique et de recyclage des héritages... Cet élargissement de la génétique explorerait des problématiques répondant aux questions que pose le spectacle de la création en général : quelles sont les stratégies qui inscrivent ces documents dans des processus d'invention, de composition et d'expression, qui parient sur le long terme et renouvellent le genre? Cette génétique engagerait aussi une réflexion sur l'interprétation des macrostructures cycliques. En effet, la connaissance des documents de genèse éclaire les œuvres qui déboussolent le lecteur lorsqu'elles excèdent des normes habituelles. Ainsi, une polémique avait opposé Claude-Edmonde Magny et René Garguilo, à propos des Thibault de Martin du Gard. La thèse de l'universitaire5, s'appuyant sur la genèse du roman, était une réponse à la critique de Claude-Edmonde Magny dans son Histoire du roman français depuis 1918. Elle avait perçu une «lézarde», une grave «rupture de construction 6 » entre les deux massifs des Thibault. Une génétique des cycles, qu'il s'agisse de cycles à entrée «unique» ou à «entrée multiple», formulerait des pistes de lectures «cyclisantes», grâce à un changement d'échelle, donc de lisibilité.

Le présupposé d'une telle génétique est celui-ci : le fait que les genèses additionnées des œuvres, prises les unes sans les autres, disent peu de la création de l'ensemble, qu'un accès à la genèse globale éclaire. Les perles font le collier mais le collier est plus que la somme des perles. Les créateurs regrettent d'ailleurs les jugements qui n'ont pas le souci de replacer le roman dans l'ensemble du cycle ou dans le projet d'une symétrie longitudinale 7 , qu'envisage un Jules Romains. Zola, qui nomme l'arbre généalogique des Rougon-Macquart son «cadre régulateur» dans la préface 8 d'Une page d'amour, l'édite après le scandale de L'Assommoir, qui fait ombrage au projet cyclique fondé en raison. D'autres écrivains souhaiteraient différer les publications afin de livrer l'ensemble en une fois, tel Proust, qui espérait, jusqu'en 1920, faire paraître la suite et la fin de La Recherche «d'un seul coup, de façon que tout s'explique et se justifie», «[...] pour que le lecteur puisse [le] juger sur l'ensemble ${ }^{9} »$. Une génétique des cycles respecterait le souhait des écrivains de voir leur œuvre reconnue dans leur intégrité organique. Ce besoin de reconnaissance peut même tourner au fantasme et remonter dans les fictions, sinon dans le paratexte (préfaces, correspondance, entretiens...). En effet, la découverte de l'idée cyclique est parfois thématisée par les écrivains sous une forme dramatique ou lyrique, de sorte que fabuler sur «l'accouchement du cycle», de façon directe ou métaphorique,

\footnotetext{
5. R. Garguilo, La Genèse des «Thibault» de Roger Martin du Gard. Le problème de la rupture de construction entre «La Mort du père » et «L'Été 1914 », Paris, Klincksieck, 1974.

6. Voir C.-E. Magny, Histoire du roman français depuis 1918, Paris, Éditions du Seuil, 1971 [1950], «Le ciment des Thibault : sa lézarde», p. 280-284.

7. J. Romains, Les Dossiers préparatoires des «Hommes de bonne volonté», op. cit., p. 52.

8. E. Zola, Préface d'Une page d'amour, dans Les Rougon-Macquart, éd. Henri Mitterand, Paris, Gallimard, coll. «Bibliothèque de la Pléiade», t. II.

9. Correspondance de Marcel Proust, éd. Ph. Kolb, Paris, Plon, 21 t. (1970-1993), respectivement, t. XV, p. 133 et t. XIX, p. 759
} 
constitue une sorte d'épisode clé de la figuration a posteriori de l'histoire intime du projet ambitieux que l'écrivain rapporte. Se construit la scénographie d'un eurêka! cyclique que Zola met en abyme, à divers moments du cycle des Rougon-Macquart : vision prophétique puis rétrospective de Pascal-Zola, entre La Fortune des Rougon et Le Docteur Pascal, vision artistique de l'écrivain Sandoz, porté par son idée, tout en craignant l'écrasement :

Je vais prendre une famille, et j'en étudierai les membres, un à un, d'où ils viennent, où ils vont, comment ils réagissent les uns sur les autres; enfin, une humanité en petit, la façon dont l'humanité pousse et se comporte... D'autre part, je mettrai mes bonshommes dans une période historique déterminée, ce qui me donnera le milieu et les circonstances, un morceau d'histoire... Hein? tu comprends, une série de bouquins, quinze, vingt bouquins, des épisodes qui se tiendront, tout en ayant chacun son cadre à part, une suite de romans à me bâtir une maison pour mes vieux jours, s'ils ne m'écrasent pas ${ }^{10}$ !

Autre argument en faveur d'une génétique des cycles : elle serait à la mesure du XXIe siècle qui développe le tout-numérique. En effet, la technologie offre des instruments puissants pour éditer les cycles sur la Toile. Des perspectives innovantes, relatives aux modes de lecture, se dessinent avec les cycles réénoncés digitalement. Les lire en tous sens, et à toutes les vitesses - autoroutes ou sentiers buissonniers - en se libérant de la linéarité chaîniste. Un écrivain comme Daniel de Roulet a saisi les potentialités du numérique pour éditorialiser $L a$ Simulation humaine, qui s'étale de 1938 à 2013. Son roman-fleuve «digitalisé » retrace, en deux cent quatre-vingt-dix-sept chapitres, la vie de deux familles, l'une japonaise, l'autre européenne, prises dans l'aventure du nucléaire d'Hiroshima à Fukushima. Une application gratuite et transmédia permet d'en mener des lectures variables, selon un menu proportionnel aux appétits du lecteur : «un avant-goût (5 minutes), une nouvelle (1 heure), un roman (2-5 heures) ou tout un roman-fleuve inédit (50 heures) ${ }^{11} \gg$ :

[...] dans cette première phase du projet qui sera disponible publiquement en avril 2014,j'ai procédé pour mon lecteur comme si j'étais un libraire à qui il vient demander conseil. Entrant dans ma librairie (mon corpus), il commence par feuilleter les livres exposés sur les tables pour en avoir un avant-goût. Ensuite, il demande au libraire de lui conseiller un texte court, une nouvelle, par exemple, quatre à dix chapitres à lire en moins d'une heure. Ou bien il demande un vrai roman, de la lecture pour deux à cinq heures au plus, mais pas les cinquante heures qu'il lui faudrait probablement pour lire le corpus entier. Je me suis donc fixé de proposer à mon lecteur six avant-goûts ou six nouvelles ou trois romans ou le corpus en entier. Quand il entre dans l'application, disponible gratuitement sur téléphone, tablette et ordinateur, le lecteur peut donc choisir comme chez un libraire.

Si les corpus anciens numérisés constituent un capital culturel, il faudrait réciproquement, ne pas se priver de tirer profit de la reconfiguration que proposent les humanités numériques. Cet exemple d'application gratuite pourrait être imaginé pour les Rougon-Macquart de Zola, La Comédie humaine de Balzac, Jean-Christophe de Romain Rolland, etc.

Tant par rapport à l'épistémologie de la génétique, que par rapport au numérique, une réflexion sur les corpus cycliques serait donc dans l'ère du temps et aborderait autrement la genèse de ces monuments de papier des XIX et XXe siècles, supposant des outils d'analyse

10. E. Zola, L'Euvre, dans Les Rougon-Macquart, op. cit., t. IV, p. 162.

11. < www.daniel-deroulet.ch/simulation-humaine/ >, site consulté le 1er octobre 2015. 


\section{Technologies d'écriture cyclique : le ponctuel et le permanent}

appropriés. La génétique cyclique investira la langue, le texte, le corpus en proposant de jouer, sur le plan linguistique, autant que poétique et esthétique, avec la variation d'échelle, la proportionnalité et le rythme. L'exemple de Zola servira, dans un esprit comparatif avec d'autres écrivains, à illustrer cette proposition. Nous nous focaliserons, ici, en particulier sur la façon dont cette approche permet de reconsidérer à nouveaux frais la question de la «révision» au long cours des cycles.

La première exigence d'une génétique des cycles concernerait la description de la méthode de travail des écrivains. À propos de Zola, les études de genèse ont décomposé sa démarche : une Ébauche qui fournit le scénario, des fiches Personnages qui dressent l'état civil du personnel, deux Plans détaillés qui assemblent la matière, un ou deux Plans généraux assurant une vision synoptique, des croquis de lieux, des enquêtes de terrain et des Renseignements divers. La réitération que fait Zola de cette méthode pour chaque roman a conduit la génétique des années quatre-vingt à conclure à sa systématicité, sans doute trop loin des pratiques empiriques et des projets de romans qui font évoluer un style génétique en une pluralité de styles et de manières, inscrite dans la longue durée des expériences scripturales. Il était dans l'air du temps, sous l'influence de la narratologie, de dessiner l'épure fonctionnelle du dossier préparatoire, qui illustrait la distinction entre l'écriture à processus et l'écriture à programme ${ }^{12}$, dont Zola fut désigné comme le parangon. Il est dans l'air de notre époque d'être sensible aux métamorphoses de l'écriture «tout au long de la vie».

Qui plus est, la théorie se heurte à l'empirie. Un regard qui souligne les variations dans la méthode de travail modélise plus finement ce qui ressemble à des innovations que réclame la poïétique du cycle, à l'échelle d'une macrotemporalité. Les dossiers de La Fortune des Rougon et de La Curée sont moins construits, notamment au regard de l'Ébauche, qui au sens strict n'existe pas ou est nommée «Premiers détails». Les dossiers de La Conquête de Plassans et de La Faute de l'abbé Mouret sont tout aussi peu organisés. Le dispositif se stabilise à partir de Nana, neuvième roman de la série, en 1880, une date qui correspond à la publication du corps de doctrine publié dans Le Roman expérimental. L'Ébauche est parfois dédoublée dans les cycles qui suivent; l'enquête de terrain se transforme en «Voyage », à Sedan, à Lourdes et à Rome, prouvant la montée du témoignage subjectif. Les rubriques du dossier préparatoire évoluent, du début à la fin des trois grands cycles. La dialectique du ponctuel et du permanent respecterait davantage la plasticité des stratégies de création cyclique, les thèmes, les formes choisies et les enjeux de chaque série. Plus généralement, l'artiste de talent mobilise la technique sans qu'elle l'immobilise, car le choix de la forme-cycle suppose fondamentalement le mouvement d'une «marche» qui bouscule automatismes et ficelles, dont se sert l'écriture feuilletonesque d'un Ponson du Terrail. Chez Jules Romains aussi, la pratique de l'écriture est présentée comme le résultat d'un dispositif de création en devenir, une propédeutique instrumentale qui évite l'épuisement de l'écriture :

12. L. Hay, «Nouvelles notes de critique génétique : la troisième dimension de la littérature», Texte, $\mathrm{n}^{\circ} 5 / 6,1986-$ 1987. 
Et, j'exagérerais à peine en disant que, par la suite, plusieurs de mes livres n'ont été qu'une façon de me faire la main, d'éprouver tour à tour mon instrument et la matière ou encore d'explorer des territoires qu'il s'agirait un jour d'occuper et d'organiser ${ }^{13}$.

On peut souhaiter une modélisation plus proche des données empiriques en embrassant de façon plus nette la variance des dossiers et la richesse de la palette. Sur l'étendue du cycle zolien par exemple, il s'actualise en effet une instance qui régule les métamorphoses de l'instrument, afin de penser son adaptation à des besoins évolutifs en lien avec les expériences créatrices. Ce n'est pas ce «Je» qui ouvre l'Ébauche le temps d'une genèse, ni même le sujet auctorial qui inaugure la geste dans les «Manuscrits originels» de 1868 par un acte de rupture avec Balzac. C'est le «moi » stratifié sur la longue durée de l'écriture tâtonnante sur laquelle pourrait se pencher la recherche autobiographique. Car, là où la génétique des textes a décelé une instrumentalisation objective du dossier préparatoire par l'écrivain, une génétique du cycle verrait une instrumentation subjective de l'écrivain grâce au dossier préparatoire. Le romancier ne fabrique pas mécaniquement son kit d'outillage, comme l'ont martelé les critiques réduisant Zola à un «ingénieur», mais il s'incorpore à ses outils autant qu'il les incorpore et les dépasse, au profit d'une construction de l'œuvre et de soi. Celle-ci repose sur la révision permanente du dispositif, qui introduit l'antisystème dans le système, l'imaginaire et le fonds mythique dans l'art des calculs programmateurs. La variation, visà-vis d'une taxinomie pratique adossée au positivisme d'époque, sauve un écrivain comme Zola du figement, parce qu'elle souligne sa liberté créatrice qui puise dans tous les héritages sur l'étendue des trois cycles. Balzac, en dépit d'une cyclisation rétrospective fondée sur le retour des personnages, n'en conserve pas moins une liberté créatrice qui pondère les calculs de l'architecte, en jouant sur la place, le rang et le rôle dévolus à ces retours segmentés de personnages. Cette variance apparaîtra moins clairement au sein d'une génétique des textes qui pense le processus créateur dans les limites restrictives de l'œuvre individuée. Les instruments de travail tirent bénéfice d'une analyse dans la durée, au sein d'une perspective qui suppose de dépasser le cloisonnement des dossiers préparatoires des œuvres, au profit d'une génétique intra-, inter- et trans-cyclique.

Qu'ils programment l'agencement cyclique dès le début, tel Zola, ou qu'ils aient l'illumination tardive d'une cyclisation de leurs œuvres déjà produites, comme Balzac, les écrivains cycliques s'approprient, dans le domaine de l'écriture littéraire, une forme de «penser global / agir local». En effet, chaque œuvre est produite, autant qu'elle peut être lue, sous l'angle d'échelles plurielles et graduelles. Le volume en cours de fabrication est une pierre du monument, fût-il inachevé. Le volume que découvre le lecteur s'autosuffit, certes, mais il offre des possibilités inédites de décloisonnement de ses limites grâce à l'arrière-plan du chemin cyclique déjà produit avec lequel il dialogue. La cyclisation optimise une forme d'auto-engendrement de la lecture tabulaire et rhizomique, et permet à l'écrivain de jouer sur des temporalités multiples qui reconfigurent, sur l'étendue du cycle, la place flottante de chaque volume dans la série, et même, la «valeur» des matériaux, genres et styles qui le

\author{
Auto-poïétique \\ cyclique : le local \\ et le global
}

13. J. Romains, Les Hommes de bonne volonté, Paris, Flammarion, 1958, t. I, p. 5. 

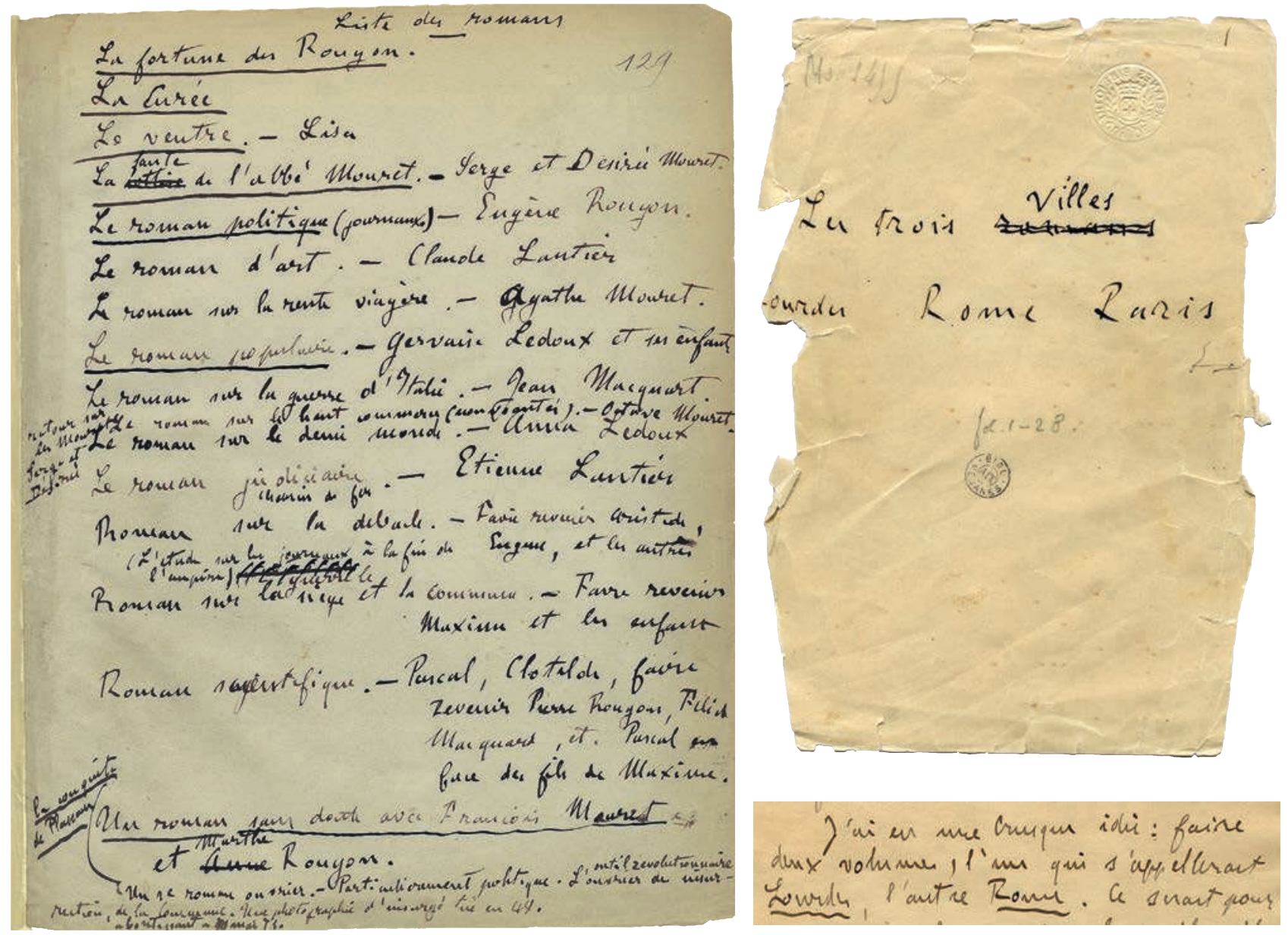

Fig. 1 (à gauche) : Émile Zola, «Liste des romans », 1872

(BnF, département

des Manuscrits,

NAF $\left.10345, f^{\circ} 129\right)$

Fig. 2 (à droite) :

«Les trois Villes/

Lourdes Rome Paris »

(Bibliothèque Méjanes,

Aix-en-Provence,

Fonds Émile Zola,

«Lourdes, plan, notes

et documents », t. I, $\mathrm{f}^{\circ} 1$ )

Fig. 3 (en bas à droite) : «J'ai eu une brusque idée : faire deux volumes,

l'un qui s'appellerait Lourdes, l'autre $\underline{\text { Rome }}$ " (Bibliothèque

Méjanes, Aix-en-Provence,

Fonds Émile Zola, «Lourdes, plan, notes et documents »,

t. I, f 60 ) constituent à l'échelle microscopique. Réceptions et genèses successives des œuvres assurent ainsi le réajustement permanent des stratégies textuelles et idéologiques de repositionnement du cycle, selon les circonstances qui déprogramment et reprogramment le projet initial : œuvre-riposte, œuvre-combat, œuvre-répit, œuvre-diversion, œuvre-charnière...

C'est la raison pour laquelle une génétique cyclique pourrait sans doute privilégier le décloisonnement des limites de l'avant-texte du roman et le dépassement d'une approche chaîniste, plus pertinente dans un genre connexe tel que la «suite». Si la narratologie a conçu avec l'intertextualité l'ouverture du texte au-delà de sa clôture, la génétique a peu envisagé les migrations entre dossiers, entre brouillons et placards corrigés, à l'échelle macro, qui se justifie pleinement dans le cas du cycle. Il est possible d'approcher l'imaginaire migratoire au cœur de la création, le travail de dissémination des motifs qui s'ensuit dans l'espace cyclique, ainsi appréhendé dans la variété des retours, qui ne se réduisent pas, loin s'en faut, aux personnages de la tradition balzacienne. L'Ébauche de Lourdes, à la charnière des deux cycles, prend appui sur les deux derniers romans de la série qui précède : «Reprendre tout dire, tout connaître, pour tout guérir du Docteur Pascal14», «Encore moins d'intrigues que dans La Débâcle ${ }^{15}$ ». À l'inverse, s'il refuse de prendre dans Lourdes un médecin à cause du Docteur Pascal, trop proche, la figure du prêtre lui paraît assez distante de La Faute de l'abbé

14. Bibliothèque nationale de France, Ms NAF 1455, Lourdes, Deuxième Ébauche, $\mathrm{f}^{\circ} 21$ 15. Ibid., f ${ }^{\circ} 155$. 
Mouret et de La Conquête de Plassans. Pour autant, le final du Docteur Pascal montrant l'enfant offrirait, à la fin de Paris, un bel écho. Les effets de contagion dépassent le couple modèle/repoussoir et relèvent plutôt de regards obliques sur des emprunts locaux, qui proposent à la fois les germes et les obstacles d'une auto-poïétique. Ces reprises montrent aussi que sortir d'une œuvre, ou d'un tome, et entrer dans un(e) autre peut s'avérer difficile en raison d'une forme d'attachement ombilical qui ramène l'écrivain vers un souvenir d'œuvre encore vif dont il faut s'affranchir afin de préserver le rythme éditorial, supposant mentalement de se couper de l'œuvre fraîchement publiée. Ainsi de l'influence de Germinal, pivot du cycle, qui pèse sur la genèse de La Terre, par-dessus L'EEuvre. Zola fait revenir le roman de 1885 dans le dossier préparatoire du roman paysan, deux ans après :

Cela serait donc taillé sur le plan de Germinal, en cinq parties, avec de courts chapitres 16 .

[...] Ce serait mieux déjà, cela ne rappellerait pas les Hennebeau ${ }^{17}$.

Se méfier du dénouement de «Germinal» et de «l'Euvre» 18 .

Ne garder que la camaraderie de Nenesse et de Delphine, mais ils ne partagent pas Olympe (Germinal) ${ }^{19}$.

Le roman de la mine, une fois «digéré», refait surface dans Les Trois Villes selon une relecture portant sur la peinture des foules : «Il faut que dans Paris, j'aie, comme dans Germinal un très grand fonds de souffrance $[\ldots]^{20}$ », pense Zola. L'originalité de ces transferts est le tangage entre l'écriture - ici et maintenant - du roman en cours, et le voyage lointain vers les origines, ou, à l'autre bout, du côté des horizons, quand ce ne sont pas des connexions avec la série de «Contes et nouvelles», ou avec la correspondance et les articles du journaliste chroniqueur, ou avec ses adaptations théâtrales, dont les échecs perturbent la chronologie du cycle. Face à l'échec du Bouton de rose au théâtre, la ferveur du public revient grâce à une œuvre osée. Le 6 mai 1878, Edmond de Goncourt décrit un dîner chez Véfour, en présence d'un Zola survolté : «"Je vais être obligé de faire Nana... Au fond, ça dégoûte les insuccès au théâtre... La Curée attendra, je vais faire du roman"... Et il continue à faire tourner son couteau ${ }^{21}$.» Cette prise en compte de la production globale plurigénérique, sur laquelle se détache le cycle, est une façon de faire l'hypothèse d'une mémoire des formes, d'un imaginaire transverse du déjà-écrit contaminant. L'écriture cyclique tend alors à rejouer et déjouer les œuvres qui précèdent au sein d'une temporalité souvent bousculée mais utile à la fabrication des «microcycles» que se plaît à construire le lecteur-fureteur : on retiendra par exemple, dans Les Rougon-Macquart, les microcycles «Tableau de Paris» (La Curée, Le Ventre de Paris, L'Assommoir...), «Portrait d'Octave Mouret» (Pot-Bouille et Au Bonheur des dames), «Grandeur et décadence d'Aristide Rougon» (La Curée et L'Argent qui le rebaptise Saccard), ou encore le microcycle d'encadrement «Hérédité» (La Fortune des Rougon et Le Docteur Pascal). En dépit de leur différence, certaines tactiques de micro-appariement rapprochent

16. Ébauche de La Terre, $\mathrm{f}^{\circ} 424$

17. Ibid., fo 489.

18. Plans détaillés de La Terre, $\mathrm{f}^{\circ} 395$.

19. Ibid., f ${ }^{\circ} 148$.

20. Ébauche de Lourdes, fos 23-24.

21. Le Journal des Goncourt. Mémoires de la vie littéraire, éd. R. Ricatte, préface et chronologie de R. Kopp, Paris, Robert Laffont, coll. «Bouquins», t. II, p. 778. 
un Zola d'un Jules Romains. Elles consistent à assurer une préparation binoculaire de deux volumes : Zola distribue le monde politique entre Son Excellence Eugène Rougon et La Débâcle, le monde ouvrier entre L'Assommoir et Germinal, tandis que Jules Romains compose, dans un même élan habituel, Prélude à Verdun et Verdun, les tomes XV et XVI des Hommes de bonne volonté. Cette stratégie stimule la marche en avant du roman selon une sérialité partielle, les deux volumes se créant l'un l'autre. L'invention au long cours suppose ainsi des écritures tabulaires dans l'espace cyclique.

Parallèlement, l'écriture interagit aussi entre les œuvres, avec l'éventail des productions concomitantes de l'écrivain, selon des finalités et des effets divers. L'écriture théâtrale et celle des «vies hérö̈ques» assurent une maturation autobiographique chez un Romain Rolland au cours de la rédaction de Jean-Christophe. L'alternance des genres, chez Zola, vise aussi une tactique éditoriale tenant le public en haleine, tout en permettant d'élargir, dans tous les cas, le laboratoire d'écriture, les expériences et les apprentissages artistiques au long cours de l'écrivain polygraphe. En somme, pas de cycle régénéré sans respiration et diversion exogènes qui supposent un temps élastique variable selon les écrivains, et, inversement, pas de cycle cohésif sans forces centripètes endogènes. Une génétique cyclique gagnera à être globalement culturelle, à penser les emboîtements des contextes déterminants.

Le classement des documents de genèse n'a alors plus la même finalité, si l'on s'appuie sur les logiques migratoires, centripètes et centrifuges, d'une constellation en mouvement. Tandis que la présence des feuillets mal classés, appartenant à d'autres dossiers, sera marginalisée par la vision monographique, une approche corrélée des dossiers et des manuscrits entre eux conduit à réfléchir à des temporalités plurielles, en particulier celles relatives à des éléments différés (scénarios, personnages, résumés et fiches de lecture...), relégués dans un autre dossier servant cependant de réservoir et déversoir à l'ensemble cyclique qui les réinjecte selon les besoins. Pour comprendre la fiche «Mai romain» glissée dans les notes de Jean-Christophe, il faut retourner au petit roman écrit à Rome en $1890^{22}$. Les logiques du rewriting s'avèrent complexes : Rolland réécrit en partie son Journal, consignant ses impressions de voyage en Allemagne, en 1896, sous la forme de fiches, réparties dans les dossiers de travail de L'Aube, Le Matin et La Révolte. Cette circulation des documents de genèse élargit la cartographie du laboratoire d'écriture. Martin du Gard, se représentant en créateur arpenteur, concentre ce processus temporel dans un cadre spatial anticipateur. En 1920, dans sa maison du Berry, composant les épisodes enchaînés des Thibault, il cartographie son projet de cycle grâce au mobilier qu'il réquisitionne pour la circonstance :

[...] j'avais rassemblé dans une grande pièce toutes les tables de la maison. [...] Enfermé du matin au soir, debout au centre de cet échiquier, avec un crayon, un bloc-notes et quelques fiches à la main, j'allais d'une table à l'autre, me ravisant, revenant dix fois sur mes pas afin de déposer enfin dans la case appropriée la note que je tenais entre les doigts. Peu à peu les fiches s'empilaient sur les tables; les dossiers se formaient. [...]. Ils étaient tous là mes personnages, présents au départ, avec leurs physionomies particulières et les traits dominants de leurs personnalités 23 !

22. Voir «Mai romain», dans B. Duchatelet, Les Débuts de Jean-Christophe (1886-1906), Service de reproduction des thèses, Lille III, 1975, t. II, p. 253-256.

23. R. Martin du Gard, Euvres complètes, Paris, Gallimard, coll. «Bibliothèque de la Pléiade», 1955, « Souvenirs », t. I, p. 81. 
En utilisant les treize tables installées dans le pavillon du Verger d'Augy24, à la manière d'une miniaturisation spatiale assurant la pré-vision panoptique de l'immensément grand, au même titre que l'arbre généalogique de Zola et le «plan unanimiste» de 1932 de Jules Romains, Martin du Gard matérialise des protocoles de composition qui sont des opérateurs de (pré-)lisibilité du cycle : l'expansion héréditaire résumée par un arbre (Zola), le pullulement organisé par des constellations (Jules Romains), l'empilement de fiches réparties dans l'espace domestique (Martin du Gard). Comme pour Zola, ce dernier se donne des «cadres ». Dans ces casiers, «alvéoles de cire», Martin du Gard rêve d'y mettre le vivant, qu'il désigne comme «son miel25». Les deux écrivains vérifieraient l'observation de Louis Desprez, à nuancer pourtant : «À l'encontre de Balzac, M. Zola construit du dehors; la symétrie minutieuse d'un architecte; tout en place d'abord; il ne s'agit plus que de remplir le cadre26. ${ }^{26}$ Les cycles, en mouvement, bousculent leur moule inaugural, même si Zola prend l'idée de cycle au sérieux. Les processus en jeu engagent à penser que la génétique pourrait mettre en avant plutôt un modèle cybernétique : dossiers poreux, autorégulation, insertions associées aux notes de régie, comme celles de La Recherche réclamant d'ouvrir les cahiers les uns sur les autres, ainsi que le recommande Claire Bustarret27, et remaniement tâtonnant des œuvres antérieures chez Balzac, afin de mieux les intégrer au cadre de La Comédie humaine. Plus encore, l'œuvre-monde balzacienne est marquée profondément par la porosité 28 . Sans doute, serait-ce le troisième intérêt d'une génétique du cycle : poser la question de l'énergie scripturale d'un grand ensemble dont l'écriture ne cesse de transformer la direction et le sens. Quand un écrivain fait le pari de la postérité et de la productivité au long cours, au moyen de quels leviers assure-t-il la cohérence et le développement «ensemblistes» de son œuvre, sans lesquels il y aurait risque d'essoufflement à court terme?

Répondre à la question suppose de dégager la trajectoire d'une démarche évolutive de l'artiste. Judith Schlanger propose de passer à une échelle de temps supérieure :

[L'invention] est un travail de réflexion qui s'installe dans la durée, qui connaît des épisodes, des réorganisations, des aspects accidentels et aussi des aspects ludiques [... $]^{29}$.

Le cycle des Rougon-Macquart, mis en place entre 1868 et 1869 , se déroule et croît à un rythme soutenu. Maintenir ainsi une force d'innovation reste un mystère. Pour y répondre, la génétique des textes a insisté sur les soliloques préparatoires de l'écrivain, sa parole performative qui ordonne : une voix démiurgique d'une grande puissance de vision et de

24. En 1907, Roger Martin du Gard avait décidé de construire son «Verger» dans le parc de la propriété de ses parents.

25. R. Martin du Gard, «Souvenirs», op. cit., p. 132

26. Jugement de L. Desprez dans E. Zola, Les Rougon-Macquart, op. cit., t. III, p. 1863.

27. Voir ce qu'écrit Claire Bustarret à propos du cloisonnement : «[...] c'est par comparaison avec d'autres cahiers [...] que les incongruités apparentes de tel collage ou de telle foliotation prendront éventuellement sens, au terme de l'exploration systématique du corpus », «Les Cahiers de La Recherche, un labyrinthe de papier?», dans Proust aux brouillons, dir. N. Mauriac Dyer et K. Yoshikawa, Turnhout, Brepols, 2011, p. 24.

28. Voir D. Massonnaud, Faire vrai. Balzac et l'invention de l'œuvre-monde, Genève, Droz, 2014, p. 265.

29. J. Schlanger, citée par B. Beugnot, «La génétique, questions de frontières », dans Critique génétique : concepts, méthodes, outils, dir. O. Anokhina et S. Pétillon, IMEC, 2009, p. 43.

\section{Méta-énonciation cyclique : le calcul et la pulsion}


scénarisation. Ont souvent été étudiés ses autocommentaires de régie et son métalangage, puisant dans les vocabulaires des arts, et pris en charge par un métadiscours qui tantôt invente,

Je voudrais, après le Rêve, faire un roman tout autre; d'abord dans le monde réel; puis sans description, sans art visible, sans effort, écrit d'une plume courante; du récit simplement; et comme sujet, un drame violent à donner cauchemar à tout Paris $[\ldots]^{30}$,

tantôt corrige et propose :

C'est cela qu'il faudra chercher, une division par parties, peut-être, qui permettra des refrains, quoique ce serait très mauvais d'interrompre le récit 31 .

L'écrivain s'appuie sur ce métalangage littéraire, qu'Henri Mitterand définit comme «la nomination des formes inventées pour les contenus et l'expression de l'œuvre en gestation 32 ». Il existe peu de rapport entre ce système zolien et sa version plus modeste, en général, chez les autres écrivains de cycles à entrée «unique». Ainsi Romain Rolland mêle quelques autocommentaires poïétiques à une importante matière rédigée sous la forme de canevas, de plans et de brouillons partiels dont les lois de construction se fabriquent a posteriori. «Dans la composition de Jean-Christophe, rien n'avait été livré au hasard [...]. La qualité artistique à laquelle je tiens le plus est celle de constructeur ${ }^{33}$.» Pourtant, les liens de causalité entre les épisodes, contrairement à Zola, ne sont pas forcément posés à l'initial du scénario. Le roman est plutôt une sorte de puzzle à constituer intuitivement à partir des pièces inventées. Le 29 juillet 1897, il révise les reliefs du roman («centre», «unité», «noyaux», «grouper», «masse mouvante», «rayon») et décline les phases de la vie de Jean-Christophe («Enfance», «Adolescence», «Jeunesse», «Virilité»...). Trois années se passent; Rolland rédige des fragments rédactionnels sur l'enfance de son héros, en alternant brouillons et notes de régie qui cadastrent et composent : «Il faut que chaque période de la vie soit comme un roman distinct, où ses caractéristiques sont groupées, concentrées, autour d'un épisode particulier ${ }^{34}$. » Il reprend son matériau, le remanie et l'organise sur les plans logiques et chronologiques. Pour autant, il n'hésite pas à écrire le «nez au vent», selon la formule de Martin du Gard, laissant proliférer les épisodes aux liens souples. Prévision, rédaction, révision s'enchaînent puis verrouillent les structures, en créant les liens de nécessité que réclame l'œuvre d'art. La relance du projet réside dans ces alternances de calcul et de pulsion scripturales, là où Zola imprime un rythme plus serré à l'image du Nulla dies sine linea. Dans chacune des genèses, la planification scénarique précède la rédaction du manuscrit sans que cette dernière ait des effets rétroactifs sur les pilotis prérédactionnels du roman. Les Plans détaillés des dossiers préparatoires ne sont ainsi jamais révisés en fonction des déviances fréquentes de la phase rédactionnelle. Pour chacun de ses romans distincts, Zola fixe le centre de la toile, en respectant le cahier des charges d'un cycle familial, dispose les premiers et arrière-plans

\footnotetext{
30. Ébauche de La Bête humaine, $\mathrm{f}^{\circ} 338$.

31. Ébauche de Lourdes, Deuxième Ébauche, fos 58-59.

32. H. Mitterand, «Le méta-texte génétique dans les Ébauches de Zola», Genesis, n 6, «Enjeux critiques », 1994 , p. 47-60.

33. Lettre à Otto Grautoff du 28 janvier 1913, cité par B. Duchatelet, Les Débuts de Jean-Christophe (1886-1906), op. cit., p. 437.

34. Ibid., p. 275.
} 
avec clarté; puis c'est le saut dans les étoiles, sans que les Plans bénéficient d'une quelconque correction. Zola s'en est libéré, comme la fusée de ses boosters. Car il faut produire, tenir le rythme, accepter l'imperfection de l'œuvre achevée, que met en scène le drame du peintre Claude Lantier, dans L'Euvre.

La rareté d'un autocommentaire soutenu, chez Rolland, s'explique sans doute par les finalités du roman-cycle à entrée «unique», vectorisé par une seule trajectoire, quand les héros de Zola s'irradient dans chaque œuvre singulière, autour desquels il faut réinventer un «monde» nouveau. Chez Rolland, il s'agit de suivre le développement d'une âme, celle de Jean-Christophe, nourrie de la vie de son auteur, au fil d'une suite de romans. De plus, son art d'écrire s'acclimate mal des excès d'une raison constructrice à la Zola, tandis que s'adjoint une durée de création plus élastique, rendant possible la révision du projet entre 1896 et 1902. Une génétique des cycles serait aussi une rythmologie comparative, attentive aux aspirations des écrivains à l'égard du temps de l'écriture.

Compte tenu de la densité du métatexte prérédactionnel zolien que souligne une approche longitudinale des dossiers de travail, le Centre Zola de l'ITEM a esquissé en 2009 un répertoire des autoconsignes de l'écrivain sous la forme d'un dictionnaire. Pour aller au-delà du classement alphabétique, la génétique devrait permettre l'étude des corrélations entre la prégenèse du cycle au sein du métadossier des «Manuscrits originels » de 1868-1869 et les dossiers préparatoires successifs. Henri Mitterand a dessiné les contours d'une approche pertinente pour les cycles planifiés : «Comment apprécier la rémanence de l'énergie génétique du programme originel?»Ce faisant, il lançait une piste aux généticiens : «Elles font au moins apparaître que la critique génétique, dans un cas comme celui-là, doit se faire elle-même dynamique, différentielle, perspectiviste ${ }^{35}$.»

Sur ce modèle, une génétique du cycle pourrait suivre le devenir de la nomenclature méta-esthétique originel. Chez un Jules Romains, elle s’énonce dans ses «Dossiers généraux », au moyen de onze grandes catégories préétablies («A. Grands thèmes conducteurs », «B. Éléments à incorporer», «C. Morceaux»...). Chez Zola, au contraire, celles-ci s'élaborent roman après roman, et restent implicites. Dès son métadossier de 1868-1869, le métalangage littéraire ne fait qu'esquisser les problématiques du rythme, de la distribution, de l'intrigue et du drame, des reliefs et des soulignements développés à chaque nouvelle genèse. Chez Romains, il apparaît, ponctuellement, un processus par lequel des notes de régie à valeur injonctive décident, à distance, des usages d'une matière ancienne, comme c'est le cas dans ses «Dossiers généraux» vers 1930: «Aller loin dans l'explication», «Éviter le ton roman $\mathrm{XIX}^{36}$ ». Le cycle à entrée unique développe donc un autocommentaire plutôt en pointillé, parce qu'il semble se situer moins du côté du ressassement que de l'épaississement qui suppose une collaboration intime avec la durée durant laquelle se construit la consistance de l'œuvre. Chez Zola, au contraire, le métalangage prérédactionnel ne cesse de se ressaisir en spirale, sur l'étendue des dossiers préparatoires, à des intervalles rapprochés de deux années en moyenne. Les occurrences du terme «Apparition», par exemple, montrent comment, au fil des Plans détaillés, ce terme s'inscrit dans des contextes approfondissant le questionnement du romancier qui «creuse» la poétique du «personnage secondaire» en tant que type, au sein

35. H. Mitterand, Zola, l'histoire et la fiction, Paris, PUF, 1990, p. 50.

36. J. Romains, Les Hommes de bonne volonté, op. cit., p. 54. 
d'un soliloque à la première personne. La démarche de Zola s'avère donc bien distincte de celle de Jules Romains : la reprise souple des «dossiers généraux » à des années de distance est une tactique des «espaces vides » à combler selon un mûrissement des matériaux au sein d'un univers mental homogène qui prend son temps, s'opposant à la densité continue des métatextes de Zola appliqués à des romans, des factures et des mondes à chaque fois nouveaux. Proust habite autrement le temps de la révision : les notes de régie, sur le recto et le verso des manuscrits de La Recherche, cohabitent avec l'activité rédactionnelle de façon fusionnelle. Ces pratiques plurielles visent cependant une même finalité propre à l'écriture cyclique : régler le jeu du calcul et de la pulsion, dans le domaine de l'invention, de la composition ou de la mémoire, qui s'appuie et se libère à la fois du déjà-écrit et du déjà-lu dans le but de fonder une auto-poïétique suffisamment énergique pour prolonger le face-à-face entre le créateur et sa création au long cours, toujours prête à l'absorber.

Par-delà le modèle fondateur balzacien, il faut remonter à Montaigne pour trouver un dispositif puissant de relecture additive, même si le rapprochement de Zola et Montaigne n'a pas le même sens que celui, plus habituel, de Montaigne et Proust : «Mes fantasies se suyvent; mais par fois c'est de loing : Et se regardent, mais d'une veuë oblique ${ }^{37}$.» Pourtant, Zola n'est-il pas au ressassement de ses opérations métatextuelles ce que Montaigne est aux textes sédimentés de ses Essais? Ils reprennent à intervalle régulier une matière antérieure qu'ils recyclent : l'un son texte sédimenté, l'autre les opérations de son métatexte prérédactionnel sur l'étendue de sa carrière. Si un écrivain de roman-fleuve peut réviser son œuvre en augmentant et ajustant ses Plans successifs à la manière d'un puzzle, un écrivain cyclique comme Zola assure cette révision à partir des opérations métatextuelles revisitées, relatives à la composition, la distribution, la mise en intrigue, les reliefs, les proportions : une sorte de répertoire poḯtique complet et évolutif à l'échelle du cycle que nourrit chaque expérience créatrice et qui permet d'orchestrer les grandes lois du genre cyclique : le retour des motifs, les résonances thématiques, la partition symphonique et fuguée du cycle. Révision cyclique :
auto-apprentissages
et feuilletés énonciatifs
Zola a lu Montaigne à 20 ans, pendant deux années durant lesquelles il fait provision de philosophie pour surmonter les découragements des années 1861-1862. Halina Suwala interprète cette rencontre en étudiant le rôle des Essais dans la formation intellectuelle de l'écrivain. Elle scrute les racines philosophiques du naturalisme qui convoque Montaigne, à côté de Michelet, Taine et Claude Bernard ${ }^{38}$. Il est possible de prolonger la comparaison des deux auteurs en remplaçant l'étude des sources par une approche génétique. Même si Montaigne est une lecture dont Mes haines se fait l'écho en 1865, Zola reste muet sur l'imagination créatrice, quand elle s'imprègne de «l'outillage intellectuel et verbal ${ }^{39}$ » d'un auteur complice. Peut-être que l'écriture de soi et la mise à l'essai de la pensée, chez Montaigne, ont toutefois marqué le jeune Zola, qui rêvait d'écrire une «Chaîne des êtres » à 20 ans, tandis qu'il dévorait justement Les Essais. Il s'agira donc d'étudier les transferts entre la technologie

37. Montaigne, Les Essais, éd. Pierre Villey, Paris, PUF, 1988, livre III, 9, p. 994.

38. H. Suwala, «Zola lecteur de Montaigne», dans Autour de Zola et du naturalisme, Paris, Champion, 1993 , p. 116. 39. Montaigne, Les Essais, éd. J. Balsamo, M. Magnien et C. Magnien-Simonin, Paris, Gallimard, coll. « Bibliothèque de la Pléiade», 2007, p. XI. 
de l'essai et la technologie du cycle, dont les opérations sont successivement revisitées. Dans les deux cas, un instrument d'investigation fabrique une connaissance nouvelle de l'écriture, antidogmatique, antirhétorique, en développement permanent. Trois opérations générales de révision peuvent être étudiées, qui font de Montaigne un modèle matriciel des écritures longues, au-delà du seul cas «Zola».

\section{La réinterprétation}

À partir de Brunetière, la philologie de la fin du XIX ${ }^{\mathrm{e}}$ siècle a orienté les éditions des Essais dans le sens d'un marquage des «couches» de textes (A, B, C), qui permettent d'identifier ses révisions dans la durée, de 1572 à 1592. Certaines «additions », chez Montaigne, ont des effets qui perturbent le sens initial. Ce sont des regards divergents que rien ne laisse prévoir et qui assurent la réinterprétation de l'idée. Le chapitre «Des pronostications » (I, XI) tourne à la satire à mesure qu'il s'enrichit : l'objet de la critique n'est plus l'inquiétude des hommes tendus vers l'avenir mais leur crédulité et la constatation de leur ignorance.

Chez Zola, la réinterprétation des opérations métatextuelles anciennes passe pareillement par le retournement, la critique, la négation qui modulent, nient ou inversent, de sorte que, comme dans les Essais, une lecture en surplomb des dossiers zoliens donne le sentiment de suivre une pensée qui «se confronte avec sa trace écrite, s'y ressaisit et y inscrit sans cesse les marques de son “progrez 40 " ». Les modalisateurs «peut-être », «à voir», «assez», «trop », dans les Ébauches et les Plans, portent des regards distanciés sur les opérations poïétiques. Par exemple, les concepts de «masse» et de «degré », touchant la densité des matériaux et la narration, subissent ce traitement. Le système des masses est respecté au début du cycle des Rougon-Macquart, fidèle en cela aux consignes des «Manuscrits originels » de 1868. Il est nuancé puis déconstruit dans Son Excellence Eugène Rougon qui répond par sa souplesse à la massivité de La Faute de l'abbé Mouret qui le précède. Au concept succède le contre-concept. La gradualité en escalier, qui marque la déchéance de Gervaise Macquart, dans L'Assommoir, se métamorphose en une gradualité fondue dans Une page d'amour à partir d'un même procédé réinterprété à distance : «Je dois étudier l'amour naissant et grandissant comme j'ai étudié l'ivrognerie, peu à peu, degré par degré41. . Ces nuances, sans lesquelles le cycle piétinerait et effacerait les résonances formelles qui le tissent, ne sont-elles pas celles de Montaigne : «J'ayme ces mots, qui amolissent et moderent la temerité de nos propositions : A l'avanture, Aucunement, Quelque, On dict, Je pense, et semblables » ? Zola ne renierait pas cette démarche «enquesteuse, non resolutive 42 ». Les Goncourt manquaient de bienveillance pour saisir cette clef montaignienne que leur donnait l'écrivain à propos du mécanisme de son talent :

Aujourd'hui, il faisait de la production incessante, bonne ou mauvaise, déclarait-il carrément, non seulement un moyen de succès, mais une espèce d'école de perfectionnement de l'écrivain. À quoi je répondais que je n'étais pas du tout de son avis, qu'il était positif que le travail donnait ce qu'il

40. A. Tournon, Montaigne. La glose et l'essai [édition revue et corrigée, précédée d'un réexamen], Paris, Champion, 2000 , p. 52.

41. Ébauche d'Une page d'amour, f 500.

42. Montaigne, Les Essais, livre III, op. cit., p. 1030. 
disait, mais non la production, que cette production du reste se faisait sans se produire chez l'écrivain de talent, ne voulant donner au public que du bon $[\ldots]^{43}$.

Chez d'autres écrivains, la stratégie de reprise permanente est différente, bien que souvent animée par la même volonté de «révision interprétative» oblique, à travers et au-delà des œuvres singulières. Chez un Balzac, c'est moins le métatexte que le texte qui fait levier : les relectures des œuvres antérieures fournissent une dynamique générative. Anthony R. Pugh a montré comment Balzac relit ses textes antérieurs comme des avant-textes : il repère dans un récit précédent des germes qui seront retraités afin d'alimenter un nouveau récit. Chaque roman terminé fonctionne comme une réserve d'idées de romans ${ }^{44}$. De son côté, Jules Romains, parce qu'il s'engage dans une logique de cycle à entrée unique, prolonge sur le plan narratif le monde unanimiste en dépôt dans la partie de la série qu'il complète, tout en révisant ses dossiers originels anciens.

\section{La généralisation}

Chez Zola, la généralisation extrait des lois de composition. Il s'agit d'un passage de l'opération à sa nomination. Les termes en -tion tels que «gradation», «progression», «disposition», «narration», «division», arrivent seulement dans les derniers dossiers de travail des Rougon-Macquart, comme si Zola se méfiait de termes trop marqués par la rhétorique pour désigner ses opérations d'écriture. Rappelons que les prépositions spatiales qui distribuent visuellement les matériaux («mettre entre», «plus loin») au début du cycle des Rougon-Macquart se fixent en un lexique abstrait à la fin («espacer», «intervalle», «échelonner 45 »). Chez Montaigne, des formules de synthèse valorisent les idées qu'une première strate indiquait légèrement. Des anecdotes prennent un statut neuf lorsque le philosophe les enrichit d'un préambule qui dégage le principe ou transpose une anecdote en observation morale. Dans «De l'exercitation» (II, 6), la strate [C] change de perspective sur la mort et tire une vision générale de l'existence, selon une philosophie de la nature, où cette mort est intégrée à la condition humaine. Comme l'explique André Tournon, ce type de variantes constitue un commentaire qui définit le sens général des propos, ébauche une nouvelle distribution des thèmes, accentue ce qui n'était qu'esquisse. Le texte de Montaigne comme le métatexte prérédactionnel de Zola reformulent les strates antérieures dans le sens d'une connaissance plus générale et abstraite, qui extrait le savoir que fournissent les expériences d'écriture : cette expertise est un atout, un auto-apprentissage, si tant est que l'expertise n'étouffe pas l'inspiration.

\section{L'objectivation}

L'écrivain conceptualise les notions expérimentées, dégageant ses lois temporaires d'écriture pour mieux les identifier comme des principes fédérateurs sans les figer en une rhétorique de conventions : «Et il faut une gradation, pour qu'il y ait composition ${ }^{46}$.» L'analogie avec

43. E. et J. de Goncourt, Journal, Paris, Laffont, t. II, p. 973.

44. Anthony R. Pugh, Balzac's Recurring characters, Toronto, University of Toronto Press, 1974.

45. Deuxième Ébauche de Lourdes, f 178.

46. Ibid., f ${ }^{\circ} 189$. 
Les Essais s'inscrit dans cette idée d'une reprise des éléments éclairés selon des rayons variés. L'expérience scripturale est retraitée selon un processus réflexif que Montaigne décrit : «Ce n'est assez de compter les experiences, il les faut poiser et assortir; et les faut avoir digerées et alambiquées, pour en tirer les raisons et conclusions qu'elles portent ${ }^{47}$.» Dans le métatexte prérédactionnel de Zola, il en va de même, puisque le métalangage à l'œuvre dans les dossiers préparatoires forme un ensemble lexical en développement, qui s'actualise, dossier après dossier, fondant ainsi une poḯtique à soi. Cette verbalisation croissante des opérations métatextuelles en boucle tend à faire évoluer des habilités ignorées en capacités conscientes d'elles-mêmes, toujours remises en jeu dans la phase rédactionnelle. «Tout lecteur de Montaigne a éprouvé l'impression de surprendre une pensée qui se reprend perpétuellement, se cherche, s'élabore à chaque page 48 », écrit Tournon. Le lecteur des manuscrits de Zola qui les lirait comme une méditation en acte le ressentirait sans doute aussi. Montaigne vit pour se connaître : "Qui ne voit que j'ay pris une route par laquelle, sans cesse et sans travail, j'iray autant qu'il y aura d'ancre et de papier au monde 49 ?», Zola a compris aussi combien il lui était nécessaire de «s'éplucher le cerveau» sur l'étendue de sa carrière, afin de fonder un naturalisme alternatif, un antisystème, une autocritique et une contre-expertise de ses automatismes incorporés, en contrepoint même du naturalisme théorique : le témoignage d'un cerveau au travail, une enquête humaine, la plus longue de sa vie, dont il figure le centre, et qui sera l'envers de ce fantasme d'un «crâne de verre». Il s'agit bien d'un crâne opaque, celui à l'origine des reprises infinies de ses actes d'écriture prérédactionnelle et rédactionnelle, afin, comme Montaigne, de pénétrer la profondeur de ses replis. En ce sens, une génétique cyclique, appliquée à la création zolienne, peut considérer que les dossiers préparatoires du romancier constituent une forme d'autobiographie intellectuelle, le journal du spectacle de la genèse, dont les romans effacent les traces au profit d'un neutralisme de la voix narrative. La pensée en ébullition, des tranches de vie cérébrale, une éthique du renouvellement de soi resteront conservées par les manuscrits.

Les écrivains cycliques que nous avons évoqués dévoilent que la genèse d'une œuvre longue a ceci de passionnant qu'à mesure qu'elle avance, le projet se modifie, s'altère, se réinvente. L'œuvre ne vient pas habiter le cycle en tant que structure préalablement dessinée, elle le crée au fil de sa croissance : tel est le pari auquel la génétique pourrait être sensible lorsqu'elle scrute ces opérations de concentration-éclatement/agrandissement-miniaturisation/ marqueterie-assemblage/morcellement-continuité/focalisation-diversion/tension-pause, qu'actualisent les poḯtiques du cycle, et qu'actualisent aussi les lecteurs dans la diachronie des réceptions culturelles. Car, de Zola à Martin du Gard, les écrivains ont aussi cette crainte que, de leur vaste cycle, il ne reste finalement que quelques extraits d'anthologie, quelques morceaux de bravoure ramenant leur ambition transcendante à la survie aléatoire des feuillets d'œuvres dans l'histoire des civilisations. Le numérique, de ce point de vue, ouvre une brèche, dont de Roulet fait une opportunité.

47. Montaigne, Les Essais, op. cit., p. 931.

48. A. Tournon, op. cit., p. 70.

49. Montaigne, Les Essais, op. cit., livre III, p. 945. 
Les écritures des grands ensembles ont une révision qui ne relève pas d'un système contrôlant l'adéquation d'une réalisation à une volonté initiale. L'écrivain, lui-même en métamorphose, loin des portraits littéraires en pied, prend, comme son œuvre, des directions en partie inconnues parce que la révision, en régime de lenteur et de longévité, est porteuse de création auto-poïétique qui inclut aussi les projets ébauchés et avortés, les spectres cycliques derrière la charpente visible. Ce sont là des pistes pédagogiques pour renouveler l'approche scolaire des écrivains au collège et au lycée. Enfin, au-delà de la place que le XIXe siècle accorde à la question du cycle, il se pourrait bien que Montaigne soit une figure tutélaire de l'écriture longue et concaténée, «inachevable » comme les Essais, selon Martin du Gard, avec ses « coins» et «ses galeries $50 »$, dira Jules Romains, et sa «lecture en zigzag $51 »$, faisant écho à la figure du «labyrinthe», qu'emprunte aussi Zola pour nommer son cycle, à l'image des mondes possibles enfantés par ces monstrueuses formes informes, qui bravent la génétique des textes à chaque carrefour, et supposent son élargissement à l'échelle des grands corpus :

Naturellement, en cours de route, nous avons rencontré, Christophe et moi, beaucoup de personnages, amis ou ennemis, que nous ne prévoyions pas, ou qui nous ont intéressés plus que nous n'ayons pensé d'avance; le monde aussi a évolué autour de nous; et aussi, nous-mêmes. Jamais nous n'avons cherché à nous enfermer dans un cadre rigide 52 .

50. J. Romains, Les Hommes de bonne volonté, op. cit., p. 240.

51. Ibid.

52. Lettre de Romain Rolland du 3 janvier 1913 à Paul Seippel. Cité par B. Duchatelet, op. cit., p. 441. 
Olivier Lumbroso est professeur en langue et littérature françaises à l'université de la Sorbonne Nouvelle-Paris 3, membre du DILTEC (Didactique des langues, des textes et des cultures) et chercheur associé du CRP19 (Centre de recherche sur les poétiques du $\mathrm{XIX}^{\mathrm{e}}$ siècle). Ses travaux portent sur Zola et le naturalisme, sur la didactique de la littérature et les humanités numériques. Au sein du projet ANR ArchiZ (2012-2015), coordonné par Alain Pagès, il a été le responsable scientifique du projet pour le partenaire ITEM, au sein de l'équipe Zola. Il est auteur et coauteur d'ouvrages portant sur le naturalisme, du point de vue génétique (Les Manuscrits et les dessins de Zola, 2002, coécrit avec Henri Mitterand; Zola. Le Signe et la Consigne, 2009, coordonné par Philippe Hamon), du point de vue poétique (Zola, La plume et le compas, 2004) et dans la perspective des apprentissages de l'écrivain (Zola autodidacte, 2013). Il est membre des comités de rédaction de Genesis et des Cahiers naturalistes.

olivier.lumbroso@univ-paris3.fr

Résumés

\section{Génétique des textes et génétique des cycles : pourquoi changer d'échelle?}

Cet article propose à la génétique des textes de réfléchir à l'étude des grands corpus manuscrits, concaténés et discontinus, laissés au cours de la genèse des «œuvres-monde» telles que les cycles, les séries et les romans-fleuves, entre le XIXe et le XXI ${ }^{\mathrm{e}}$ siècle, de Balzac à Daniel de Roulet, en passant par Zola et Martin du Gard. Le spectacle de la création étendue d'un grand ensemble, supposant des changements de taille et de forme, une élasticité de l'espacetemps de l'écriture, des circuits d'édition variés, ou encore une invention soumise aux spasmes de l'Histoire, ne suppose-t-il pas des problématisations nouvelles dans le domaine des études de genèse ? Quels concepts, démarches et méthodes sont-ils appropriés à ce changement d'échelle qui ouvre le champ des analyses macrogénétiques des corpus massifs? Le défi de l'envergure artistique ouvre des réflexions inédites sur le genre cyclique, ses héritages, ses imaginaires et ses ruptures novatrices. Il suppose aussi un nouveau regard sur la périodisation diachronique et historique, sur l'unité et la dislocation formelles des œuvres de fiction, sur le biographique et l'histoire culturelle. Les quelques propositions relatives à l'échelle cyclique, à l'autopoïétique, à la réflexivité esthétique, au projet panoptique et à sa révision identifient des aspects qui pourraient être pris en compte par le chantier nouveau de la génétique du cycle.

This article proposes to reflect on the great preserved manuscript corpora, sequential and fragmented, produced in the course of the genesis of "world-works", such as cycles, series and novel sagas, from Balzac to Daniel de Roulet, including Zola and Martin du Gard. The sight of the vast creation of a great body of documents implies modifications in size and form, flexibility of writing's space/time, a variety of publication channels, or even an invention subject to History's convulsions: Would this then not imply new issues in genetic studies? What concepts, processes and methods are appropriate for this change in scale that opens up the field of macrogenetic analyses for these massive corpora? The challenge posed by this artistic scope leads to new reflections on the cyclic genre, its legacies, imagination and innovative ruptures. It also implies a new look at diachronic and historical periodization, unity and formal dislocation of fictional works, biography and cultural history. Several suggestions regarding cyclic scale, autopoietics, esthetic reflexivity, panoptic project and its revision, identify a few aspects which could be taken into account by the new work-inprogress of the génétique du cycle.
Der Artikel schlägt der textgenetischen Methode als neues Untersuchungsfeld die umfangreichen Handschriftenkomplexe vor, die uns im Verlauf der Genese von Zyklen, Serien oder Fortsetzungsromanen zwischen dem 19. und 21. Jahrhundert überliefert wurden, von Balzac über Zola und Martin du Gard bis Daniel de Roulet. Die langfristige Arbeit an einem großen Ganzen setzt Änderungen in Umfang und Gestalt voraus, ebenso wie Elastizität der raumzeitlichen Dimension des Schreibens und verschiedene Editionsverfahren, ganz zu schweigen von einer inventio, die den Wechselfällen der Geschichte untersteht. All dies führt ohne Zweifel zu neuen Fragestellungen im Bereich der textgenetischen Kritik. Welche Konzepte, Vorgehensweisen und Methoden werden diesem Wechsel der Dimensionen gerecht, der sich aus den makrogenetischen Analysen großer Werkkomplexe ergibt? Die künstlerische Herausforderung eines solchen Projektes wirft ein neues Licht auf die Gattung der zyklischen Kompositionen, ihre Ursprünge, ihre imaginären Welten und erfindungsreichen Brüche. Sie führt auch zu neuen Einsichten, was die diachrone und historische Periodisierung betrifft, wie auch die formale Einheit und Zersplitterung der erzählerischen Werke oder ihren biographischen und kulturhistorischen Hintergrund. Die hier gemachten Vorschläge, die sich auf Maßstab, Autopoiesis, ästhetische Reflexivität des Zyklus, sein Panoptikum und dessen jeweilige Revision beziehen, benennen die Aspekte, die für das neu zu erschließende Gebiet einer „génétique“ des Zyklus zu erwägen wären

Este artículo le propone a la genética de los textos una reflexión sobre el estudio de grandes corpus manuscritos, concatenados y discontinuos, producidos durante la génesis de "obras mundo", tales como los ciclos, las series y las sagas, entre el siglo XIX y el XXI, de Balzac a Daniel de Roulet, pasando por Zola y Martín de Gard. ¿El espectáculo de la extensa creación que cubre un vasto conjunto -que supone cambios de forma y de envergadura, una elasticidad del espacio-tiempo de la escritura, circuitos de edición diversificados, al mismo tiempo que una invención sometida a los espasmos de la Historia-, no requiere acaso nuevas problematizaciones en el campo de los estudios de génesis? ¿Qué conceptos, procedimientos y métodos resultan apropiados a ese cambio de escala que abre el campo de los análisis macrogenéticos de los corpus masivos? El desafío de la envergadura artística promueve reflexiones inéditas sobre el género cíclico, sus herencias, sus 


\section{Résumés}

imaginarios y sus rupturas innovadoras. Supone también una nueva mirada sobre la periodización diacrónica e histórica, sobre la unidad y la dislocación formal de las obras de ficción, sobre la biografía y la historia cultural. Algunas propuestas relativas a la escala cíclica, a la autopoiética, a la reflexividad estética, al proyecto panóptico y a su revisión, identifican aspectos que podrían ser tomados en cuenta por el nuevo campo en construcción de la genética del ciclo.

Este artigo convida a genética textual a refletir sobre o estudo dos grandes corpora manuscritos, conectados ou descontínuos, que foram produzidos entre os sécs. XIX e XXI no decurso da génese de obras de grandes dimensões (ciclos, romances em série, romances-fleuve), desde Balzac até Daniel de Roulet, passando por Zola e Martin du Gard. O espetáculo da criação de vastos conjuntos, implicando alterações de tamanho e forma, uma elasticidade de espaço e tempo refletida na escrita, circuitos de edição diversificados, ou ainda uma invenção sujeita aos espasmos da História, não pode deixar de problematizar em novos termos os estudos de génese. Que conceitos, abordagens e métodos são mais apropriados à mudança de escala que se abre no campo da análise macrogenética desses maciços corpora? O desafio posto pela envergadura artística proporciona reflexões inéditas sobre o género cíclico, com as suas origens, imaginário e inovadoras rupturas. Igualmente pressupõe um novo olhar sobre a periodização diacrónica e histórica, sobre a unidade e a deslocação formais das obras de ficção, sobre a biografia e a história cultural. Algumas propostas relativas à escala cíclica, à auto-poiética, à reflexividade estética, ao projeto panóptico e sua revisão identificam aspectos que poderiam ser tidos em conta no novo estaleiro da genética do ciclo.

L'articolo propone alla genetica dei testi una riflessione sullo studio dei grandi corpus manoscritti, concatenati e discontinui, delle "opere-mondo" come i cicli, le serie e i romanzi-fiume tra XIX e XX secolo, da Balzac a Daniel de Roulet, passando per Zola e Martin du Gard. Lo spettacolo della creazione ampia di un grande insieme, che prevede cambiamenti di dimensione e di forma, un'elasticità dello spazio-tempo della scrittura, circuiti di edizione diversi, o ancora un'invenzione sottomessa agli spasmi della Storia, non presuppone forse nuove problematizzazioni nel campo degli studi di genetica? Quali concetti, approcci e metodi possono adeguarsi a questo cambiamento di scala che apre il campo delle analisi macrogenetiche di corpus enormi? La sfida di una tale dimensione artistica apre riflessioni inedite sul genere del ciclo, sulle influenze, gli immaginari e le rotture innovatrici. Presuppone anche uno sguardo nuovo sulla periodizzazione diacronica e storica, sull'unità e la disarticolazione formali delle opere di finzione, sulla biografia e la storia culturale. Alcune proposte relative alla scala ciclica, all'autopoietica, alla riflessività estetica, al progetto panottico ed alla sua revisione, individuano aspetti che potrebbero essere presi in considerazione dal nuovo cantiere della genetica del ciclo. 